\title{
Professional development in the next decade: Supporting opportunities in all career paths and life events
}

\section{Primary Authors}

Ryan Watkins, Planetary Science Institute, rclegg-watkins@psi.edu

Nicolle Zellner, Albion College, Albion, MI 49224, nzellner@albion.edu, 517-629-0465.

Maggie McAdam, NASA Ames Research Center. maggie.mcadam@nasa.gov

\section{Co-authors}

Nicole Whelley, University of Maryland College Park and NASA Goddard Space Flight Center Ingrid Daubar, Brown University

Christine Hartzell, University of Maryland

Kat Gardner-Vandy, Oklahoma State University

This white paper is submitted as part of a collaborative effort organized by the Equity, Diversity, and Inclusion Working Group (EDIWG), a cross Assessment Group (AG) committee.

Signatory list (55+): Google spreadsheet

\section{Synopsis + Recommendations}

Planetary Science and Astrobiology need more than numerical diversity to create and sustain a thriving scientific discipline and community; they also need scientists from a variety of backgrounds and institutions. Support is particularly needed for caregivers, people who breastfeed, people who work at Primarily Undergraduate Institutions (PUIs) and Minority Serving Institutions (MSIs), and people who choose career paths that do not include research at large universities or NASA centers. By providing supportive work and conference environments, as well as opportunities for scientists to develop skills and collaborations that will further their careers, the fields of Planetary Science and Astrobiology will become more just and equitable. Retaining people with diverse backgrounds, perspectives, home situations, and employment institutions supports our community and improves the science that is done, leading to new ideas and more creative problem solving (e.g., [1]). We make the following recommendations:

- Coordinate professional development efforts with those identified by the Astronomy and Astrophysics communities (i.e., Astro2020 recommendations);

- Initiate funding and support for faculty/researchers in nontraditional paths, including caregivers; faculty at PUIs and MSIs; scientists on non-research career paths; and grantsupported researchers.

- Provide additional support for professional development (e.g., proposal writing and review, manuscript review, classroom management); and

- Establish programs that partner faculty at PUIs and MSIs with researchers at larger institutions or NASA centers or with postdocs who seek careers in teaching. 


\section{Introduction}

Advances in the fields of Planetary Science and Astrobiology benefit when the number and reach of scientific ideas and results are maximized. These fields must welcome and support scientists who have taken a variety of paths in their careers, whether by choice or by circumstance. Opportunities to advance in these careers and to be active members of the fields must be expanded, so that more scientists are invited to give talks, to co-author review papers, to participate on missions, and to contribute to the science undertaken by the Solar System Exploration Research Virtual Institute (SSERVI) and Research Coordination Network (RCN) teams. Challenges to being active researchers in these fields result from caregiving or childcare responsibilities, institutions of employment (and their policies), systemic biases and discrimination, and other barriers to success. In this White Paper, we focus on how caregiving responsibilities and employment constraints provide challenging situations that can affect how actively scientists can participate as members of the Planetary Science and Astrobiology research communities; additional barriers are discussed in separate White Papers (e.g., [2,3]).

\section{Summary of Astro 2020 WPs}

Thirty-five White Papers related to the state of the profession were submitted to the Astro 2020 Decadal Survey, and fully half of them focused specifically on issues of education and professional development. Several of those papers suggested that federal agencies should fund research and programs that explore and implement strategies for improving the experiences of scientists (e.g., [4-7]) including primary caregivers [8]. Others advocated for funded workforce studies so that issues could be better understood and policies emplaced to address them (e.g., $[9,10])$. Still other white papers commented on the need to better support instructors at teachingintensive institutions (e.g., [11,12]), including minority-serving institutions (e.g., [13]). Among calls to improve or reform graduate education in general (e.g., [14-16]) were calls to better support faculty interested in astronomy education research and support efforts to improve education in general [17]. All of these issues (and more) are also relevant to the Planetary Science and Astrobiology communities and herein we include additional evidence to support these viewpoints.

Recommendation: Coordinate professional development efforts with those recommended by members of the Astronomy community in the Astro2020 Decadal Survey white papers.

\section{Supporting ALL Scientists in the Fields of Planetary Science and Astrobiology Mothers/Parents/Caregivers}

A recent study showed that $42 \%$ of mothers and $15 \%$ of fathers in the US leave full-time STEM (science, technology, engineering, mathematics) employment within three years of having children, although the specific underlying causes remain unclear [18]. Juggling parenting duties with work duties often makes it more difficult for parents (and especially mothers) to serve on panels, committees, and conduct field work, among other essential scientific activities like writing proposals for funding. The high costs of childcare (or lack of thereof) preferentially push women into part-time roles or out of the labor force (e.g., [19]). Mothers are much more likely than fathers to turn down career-progression opportunities that involve travelling, such as conference invitations, review panels, and field work, because of family responsibilities or out of medical necessity, especially during pregnancy or early childhood. Conferences, review panels, and committee meetings often last a week at a time, forcing parents to find and pay for childcare and other household work above and beyond the normal 9-5 workday hours. This is amplified for 
single parents who do not have a partner at home who can take on the extra childcare duties while they are on travel. Field work often requires more than a week of time, making it difficult or impossible for parents to participate.

Conferences are a major activity in the fields of planetary science and astrobiology - these are where science is disseminated, networking occurs, and many researchers find new career opportunities and develop research collaborations. The lack of childcare at many major planetary science conferences can greatly hinder the ability of parents, especially for situations where both partners are scientists, to attend and participate. This is especially detrimental for early career scientists, such as graduate students, with children, as networking is crucial for finding positions and paving their career paths. Additionally, many supervisors and committees often assume women with children are unavailable due to childcare duties, so they do not extend invitations to attend meetings, serve on panels/committees, attend networking events, etc. Even in situations where childcare is offered, it is expensive, and if childcare is not extended into the evening hours, parents often choose to spend evenings looking after children rather than networking with colleagues in informal settings. Conferences could become more inclusive by reimaging how they are planned and operated. Including time during normal working hours for networking/informal interaction would allow flexibility for people, especially parents or caregivers, who may want to be otherwise occupied in the evenings. Including recorded talks may similarly help support caregivers of all types and other people that traditional conferences do not well-serve by giving conference goers more flexibility.

Similarly, conference travel is also challenging for researchers serving in other family caregiving roles (e.g., eldercare or care for a sick partner/relative). As with small children, caring for sick or elderly adults often means around-the-clock supervision, administering medication, and assisting with everyday activities (e.g. toileting, showering, and feeding). Additionally, the need for caregiving may appear unexpectedly, due to a sudden deterioration in health or an accident, making it difficult to plan for career flexibility. Further, caregiving responsibilities disproportionately fall to women: $66 \%$ of caregivers are female [20]. While caregiving responsibilities can fall upon people at any age, especially women, the impact of societal expectations around caregiving is even more pronounced for mid-career and senior women [21]. The caregiving disparity may become more apparent as more women are active in the field at midcareer and senior levels. Efforts to increase accessibility of conferences for those with caregiving responsibilities should consider the care of dependent adults as well as minor children.

Many planetary science meetings--the meeting of the American Geophysical Union, the annual meeting of the Geological Society of America, the meetings of the American Astronomical Society (AAS), and the meeting of the AAS Division for Planetary Science, for example--provide on-site childcare or funding to offset the cost of off-site childcare, but unfortunately this funding is not currently a community-wide offering. Smaller meetings that are arguably just as important for career progression, e.g., review panels and mission team meetings, are too small to offer any kind of childcare. To offset inconsistent childcare support at meetings, as well as support other caregiving responsibilities, institutions and federal grant agencies should allow caregivers to charge childcare or other caregiving expenses to institutional budgets or to federal grants. Two national fellowships ${ }^{1,2}$ allow for childcare expenses, and serve as good examples on which NASA

\footnotetext{
${ }^{1} 51$ Pegasi b Fellowship (Heising- Simons Foundation), https://www.hsfoundation.org/programs/science/51-pegasib-fellowship/, accessed 8/17/20;

${ }^{2}$ AAUW American Fellowship, https://www.aauw.org/resources/programs/fellowships-grants/currentopportunities/american/postdoctoral-research-leave-fellowships/, accessed 8/17/20.
} 
can model similar programs for its postdoctoral and graduate student research programs and ROSES grants.

Support for nursing parents is also greatly lacking at institutions, conferences,fieldwork sites, and committee and panel meetings (e.g., [22]). While many organizations and meetings have made great strides to be more accomodating, nursing people often find themselves without a private space to nurse/pump, and without adequate resources for storing breastmilk (i.e., refrigerators, storage space, resources for transporting milk, etc.). Furthermore, the amount of time required for expressing breastmilk can be significant (often 20-30 minute blocks of time, every 23 hours; this can be as much as a 0.20 FTE job!). Notwithstanding the strides made to provide physical space to pump, organizations and events may still not provide adequate time to do these activities, nor, depending on institution, are nursing people in hourly positions allowed to charge work time for pumping (despite legal requirements in some cases ${ }^{3}$ ). Pumping during work hours is an essential medical activity that is non-negotiable for many people who nurse. Without the ability to express breastmilk, nursing people can experience physical discomfort and even medical complications if pumping time is not sufficiently accommodated. All meetings should be required to provide mothers a clean, private location for pumping and resources for storing and/or transporting breastmilk.

Time taken off for maternity leave (which varies depending on institution, career stage, and location of residence ${ }^{4}$, and can be inadequate or nonexistent), parental leave, leave for the care of sick or elderly relation(s), and/or infertility treatments can often create a publication or grantwriting gap for people, especially for parents and more so for mothers. Furthermore, our system has no way to account for these gaps. The short, often unpaid, time allotted for maternity leave often forces mothers back into their careers when they are not yet physically, mentally, or emotionally ready to do so. Parental leave for domestic partners and fathers is nonexistent at many institutions, yet it is crucially important that fathers are also given time off to bond with a new child and support their partner. All families who adopt or use a surrogate also need resources and support as they transition into life with a new child. Finally, the pressures of academic and professional cultures often discourage women from having children until they are tenured and more "able" to take time away from obtaining funding, writing papers, and contributing in other ways that serve to demonstrate their worth in the community. New measures to acknowledge and accommodate 'publication gaps' are necessary to create a more inclusive community. Furthermore, longer periods of paid leave for any/all parents and caregivers are also essential for supporting all scientists in the field.

\section{Recommendations:}

- Reimagine conferences to better serve caregivers and nursing parents (e.g., recorded talks, networking opportunities during "workday hours").

- Allow caregiving to be charged on grants and included in institutional budgets for federally competed funding.

- Improve methods to acknowledge and accommodate 'publication gaps' for people engaged with childcare and/or eldercare during promotion and hiring.

- Provide longer periods of paid family leave for all adults who provide care to other family members (including children and parents).

\footnotetext{
3 https://www.dir.ca.gov/dlse/Lactation_Accommodation.htm, access 8/31/2020.

${ }^{4}$ Fact Sheet \#28, https://www.dol.gov/agencies/whd/fact-sheets/28-fmla, accessed 8/18/20.
} 


\section{Faculty@PUIs and MSIs}

Faculty at primarily undergraduate institutions (PUIs) are well-practiced in the challenges of being excellent teachers; providing service to the college (e.g., advising, assessment); attempting to acquire, maintain, and manage (federally) funded research programs; and balancing all of that with a personal life. However, anecdotally, many faculty whose primary area of study is Planetary Science find themselves siloed in their host departments, and conversations with peers at similar institutions are few and far between. Though less than 50\% of members of the Planetary Science community report engaging in teaching of any kind, almost $20 \%$ spend the majority of their time teaching ${ }^{5}$. This percentage may seem low, but it is significant as these faculty are part of the broader "Astronomy 101" teaching community that annually reaches well over 300,000 students in introductory courses focused on the space sciences [23].

As a result of a career that takes the scientist out of the lab and into the classroom more often than not, access to networking opportunities (e.g., regular conference participation, invited talks) may be infrequent. This does not mean, however, that the PUI faculty member has little or no interest in research. Unfortunately, as a result of limited networking, though, opportunities for faculty at PUIs to conduct research, to be included in large-scale research collaborations, and/or to participate in high-level NASA-sponsored activities (e.g., missions, advisory committees) are rare. Faculty at PUIs are also significantly underrepresented on Decadal panels and committees (e.g., [12]). Diversity in institution type should be valued by NASA as much as any other axis of diversity. The direct contact that instructors at PUIs have to undergraduate students is one way for NASA to enhance outreach and education activities, especially via collaborations that exist in the SSERVIs and RCNs. As noted by [11], large scientific projects and research collaborations should recognize and better utilize the high numbers of research-trained faculty at teaching institutions; they are a valuable resource because of the insights they can bring to research and because of their access to a diverse pool of early-career scientists.

Challenges to faculty at minority-serving institutions (MSIs) are similar to those at PUIs and are compounded as a result of lower numbers of faculty in general and potentially more limited opportunities to network due to smaller amounts of federal funding (e.g., ACE Brief ${ }^{6}$ ). However, because MSIs educate a substantial fraction of the country's underrepresented minority (URM) students, providing focused and intentional networking and funding opportunities for faculty at MSIs would help to strengthen and increase diversity in the fields of Planetary Science and Astrobiology. With sustained and meaningful access to research opportunities and broader professional networks, there would also be the potential to increase the number of URM undergraduates in STEM fields [13].

To earn tenure and promotion, most faculty at PUIs and MSIs are expected to have research programs that involve undergraduate students throughout the research process. Students benefit from learning experiences supervised by research-active faculty, experiences that can be translated into undergraduate research projects and even careers in Planetary Science and Astrobiology. At the very least, these research experiences, including conference travel, can increase student enthusiasm and support for space science and exploration in general [24].

While there are several MSI-targeted programs, there are very few federal PUI-targeted funding opportunities or programs. A targeted PUI/MSI mentoring, training, and research program

\footnotetext{
${ }^{5}$ DPS 2020 Workforce Study, https://dps.aas.org/reports, accessed 8/2/20.

${ }^{6}$ https://www.acenet.edu/News-Room/Pages/ACE-Brief-Illustrates-HBCU-Funding-Inequities.aspx accessed $8 / 31 / 2020$.
} 
that pairs faculty at these institutions with faculty and scientists at research institutions or NASA centers would allow PUI/MSI faculty and their students to be intentionally involved in space science research, including Planetary Science and Astrobiology. An example of such a networking program is the Astrobiology Faculty Diversity Program (formerly known as the Minority Institution Research Support (MIRS) Program) ${ }^{7}$, which existed from 2002 - 2017. In this program, a faculty member at an MSI was paired with an investigator funded by the NASA Astrobiology program. The goal of the program was "to help train a new generation of researchers in astrobiology and to increase diversity within the astrobiology community". Dozens of URM scientists participated over the life of the program; a summary of this program is presented in [25]. A similar program could be expanded to include Planetary Science along with Astrobiology. Another example of an effective partnership specifically between research institutions is the Strategic University Research Partnership $\left(\mathrm{SURP}^{8}\right)$ that supports partnerships between JPL researchers and R1 academic institutions. This type of program could be designed to specifically partner researchers at JPL with those at MSIs and PUIs.

\section{Supporting and Embracing Many Career Paths}

Graduate education in planetary science and astrobiology is implicitly focused on one particular career path that generally includes active research. The path most emphasized is highly competitive and limited in scope: it features one or more postdoctoral appointments followed by a permanent position as a faculty member or as a research scientist at a federal agency (e.g., National Laboratory, NASA, USGS, etc.). These positions and milestones usually require winning competitive federal funding and publishing many papers. The cultural value that is placed on this career path limits the perceived benefits of obtaining higher degrees in Planetary Science and Astrobiology for early career scientists and can create unsupportive environments for those who choose or are forced into career paths that vary from the culturally expected one. Indeed, it is commonly perceived as a failure if this very particular career path is not followed.

Career paths that deviate from conducting research in secure, permanent positions must be seen and appreciated for their crucial contributions to creating a thriving scientific community. Alternate pathways include working in science education (encouraging and inspiring young people in school), science communication and public engagement, science writing and editing (a crucial activity for getting science published), soft-money research, mission operations, working in related industries, and many other activities that are valid and important contributions to the field. Scientists have a wide variety of skills, talents and interests that may lead them to participate in a career that does not include active research. It is imperative that we implicitly and explicitly stop framing these paths as 'failures' and instead find new ways to support and encourage scientists to explore many career paths so that they can contribute their set of professional skills to achieve success inside and outside of academia (as noted by [9]).

\section{Recommendations: \\ - Initiate funding and support for faculty/researchers in the field of Planetary Science and/or Astrobiology who have taken non-traditional paths, including \\ $\circ$ faculty at PUIs and MSIs; \\ $\bigcirc$ soft-money researchers; and}

\footnotetext{
${ }^{7}$ Astrobiology Faculty Diversity Program, https://astrobiology.nasa.gov/nai/funding/afdp.1.html, accessed $8 / 12 / 20$.

${ }^{8}$ Strategic University Research Partnerships, https://surp.jpl.nasa.gov, accessed 8/29/20.
} 
- scientists on non-research career paths (e.g., science education, outreach, mission operations, or other important activities).

\section{General Professional Development}

Promoting and supporting education programs focused on professional skills would strengthen and diversify the Planetary Science and Astrobiology workforces. Funded workshops focused on improving skills necessary in our community (e.g., proposal writing, proposal review, manuscript review, and pro-social skills such as bystander intervention) could substantially support or increase continued participation in the field. Through funded workshops, early career scientists from PUIs, MSIs, or universities with lower levels of grant funding could access this cultural knowledge and develop these skills to retain them in science. Funded workshops could also focus on professional development (e.g., teaching and classroom management; preparing for job interviews) and other programs to retain scientists and scientists who are faculty.

Scientists in the early stages of their career would benefit from a federally funded teaching postdoctoral program so that they could experience both teaching and research to test both options before deciding if either (or both) would be suitable to pursue as a career. Faculty at PUIs and MSIs mentor and are generally in contact with a lot of students who are underrepresented in STEM. Providing training in effective and ever-changing pedagogical techniques and methods for professors who also desire active research programs at these institutions would strengthen the pipeline of students with a diversity of experiences and backgrounds into STEM careers. A federally funded program similar to the NASA Postdoctoral Program ${ }^{9}$ could match a postdoctoral scientist who is interested in teaching with a faculty member who teaches (e.g., at a PUI or MSI). Ideally the two would conduct research in the same (or a similar) field so that a research collaboration would also emerge.

Funding for service work should also be implemented. As noted by [26], "[s]ervice work is required to keep science moving and improving". They recommend that NASA compensate everyone who reviews proposals, including external reviewers, in fair and consistent ways. Incentivizing people to accept currently uncompensated service work would diversify decisions, conclusions, or recommendations and is considered a best practice (e.g., [27]).

\section{Recommendations:}

- Provide additional support for training programs that reach a variety of scientists at different institutions (e.g., proposal writing, budget development, curriculum development and classroom management, and manuscript review).

- Establish programs that allow teaching faculty at PUIs and MSIs to partner with researchers at larger institutions or NASA centers or to partner with postdocs who would like to learn how to be effective college instructors.

\section{Conclusions}

A variety of examples and solutions have been presented that can help to strengthen the Planetary Science and Astrobiology workforces and to retain a diversity of scientists in both fields. The metrics currently used to evaluate candidates for job opportunities, career promotion, and/or recognition, including publication records, number of grants funded, and service (e.g., review panels, reviewing papers) are measures that allow societal biases to cull people from the workforce

\footnotetext{
${ }^{9}$ NASA Postdoctoral Program, https://npp.usra.edu/, accessed 8/12/20.
} 
or candidate pool or otherwise create uncomfortable or hostile work environments from which scientists feel they must leave. By providing supportive work and conference environments, as well as opportunities for scientists to develop skills and collaborations that will further their careers, the fields of Planetary Science and Astrobiology will be improved and become more just and equitable for all.

\section{References}

[1] Prockter L. et al. (2017) The Value of Participating Scientist Programs to NASA's Planetary Science Division. [2] Schmidt B. et al. (2020) Diversity in Action: Solutions for a more diverse and inclusive decade of planetary science and astrobiology, Planetary and Astrobiology Decadal White Paper. [3] Rathurn J. et al (2020a) Enabling the Planetary Workforce to do the best science by funding work that is a service to the Profession. Planetary and Astrobiology Decadal White Paper. [4] McConnell N. et al. (2019) Preparing an Inclusive Astronomy Community through Effective Professional Development, Astro2020 White Paper. [5] Moravec E. et al. (2019) The Early Career Perspective on the Coming Decade, Astrophysics Career Paths, and the Decadal Survey Process, Astro2020 White Paper. [6] Norman D. et al. (2019) Tying Research Funding to Progress on Inclusion. Astro2020 White Paper. [7] Plavcha P. et al. (2019) A partial solution to the "Postdoc Crisis" is needed. Astro2020 White Paper. [8] Zellner N. et al. (2019) Findings and Recommendations from the American Astronomical Society (AAS) Committee on the Status of Women in Astronomy: Advancing the Career Development of Women in Astronomy. Astro2020 White Paper. [9] Momcheva I. et al. (2019) Long-term Trends in the Astronomical Workforce: Analysis and Recommendations Based on the Publication Histories of 10,000 US Astronomy PhD Recipients. Astro2020 White Paper. [10] Centrella J. et al. (2019) Leadership and Participation in NASA's Astrophysics ExplorerClass Missions Astro2020 State of the Profession Considerations White Paper. Astro2020 White Paper. [11] Koopman R. et al. (2019) Integrating Undergraduate Research and Faculty Development in a Legacy Astronomy Research Project, Astro2020 White Paper. [12] Ribaudo J. et al.(2019) Primarily Undergraduate Institutions and the Astronomy Community. Astro2020 White Paper. [13] Baker, A. et al., (2019) Astronomy Faculty Development at Minority-Serving Institutions, Astro2020 White Paper. [14] Burgasser, A. et al. (2019) It's Time to Eliminate the GRE and PGRE in All Astronomy \& Astrophysics PhD Programs: Motivation, Implementation and Outcomes, Astro2020 White Paper. [15] Lund M. et al. (2019) Enabling Terminal Master's Degrees as a Step Towards a Ph.D., Astro2020 White Paper.. [16] Moro-Martin A. (2019) "Mind the gap": a call to redesign astronomy graduate education. Astro2020 White Paper. [17] Coble K. et al. (2019) The Importance of Supporting Astronomy Education Research, Curriculum Reform, and Professional Development in Astronomy Education, Astro2020 White Paper. [18] Cech E. A. \& BlairLoy M. (2019). The changing career trajectories of new parents in STEM. Proceedings of the National Academy of Sciences, 116(10), 4182-4187. [19] Rose J. et al. (2011) Women and part-time employment: Easing or squeezing time pressure?, J. Sociology. [20] National Alliance for Caregiving. Caregiving in the U.S. 2009. Washington, DC: AARP Research, December 2009. https://doi.org/10.26419/res.00062.001. [21] De Marco O. (2019) From young to youthful - the challenges of mid-career, accessed 8/30/20. [22] Calisi R. M. (2018). Got milk, must conference. Science, 359, pp. 838. [23] French R.S. (2019) Everyone's Universe: Teaching Astronomy in Community Colleges, IOP Publishing. [24] Zellner N. E. B. (2020) Advancing the landscape of Planetary Science at small colleges: Creating a community of learners, Geological Society of America Meeting, ID\# 359247. [25] Gary T. et al. (2009) The NASA Astrobiology Institute-Minority Institution Research Support Program: Strengthening the Astrobiology Community. In Bioastronomy 2007: Molecules, Microbes and Extraterrestrial Life (Vol. 420, p. 477). [26] Rathbun J. et al. (2020b) Who is Missing in Planetary Science?: Strategic Recommendations to Improve the Diversity of the Field, Planetary and Astrobiology Decadal White Paper. [27] Bennett K. et al. (2020) Preventing Harassment in Science, Workshop. 